\title{
Treatment and outcome of tenosynovial giant cell tumor/ pigmented villonodular synovitis patients receiving radiotherapy in Taiwan: a single-center experience
}

\author{
Chi-Shuo Lin $^{1} \wedge$, Yu-Mei Kang ${ }^{1,2,3}$, Keng-Li Lan ${ }^{1,4}$, Ling-Wei Wang ${ }^{1,2}$, Yu-Ming Liu ${ }^{1,2}$, Pin-I Huang ${ }^{1,2}$, Yu- \\ Wen $\mathrm{Hu}^{1,2}$, I-Chun Lai ${ }^{1,2}$, Yuan-Hung Wu ${ }^{1,2}$, Tzu-Yu Lai ${ }^{1,2}$, Wan-Chin Yang ${ }^{1}$, Jia-Cheng Lee ${ }^{1}$, \\ Cheng-Ying Shiau ${ }^{1}$ \\ ${ }^{1}$ Division of Radiation Oncology, Department of Oncology, Taipei Veterans General Hospital, Taipei, Taiwan; ${ }^{2}$ Faculty of Medicine, National Yang- \\ Ming University, Taipei, Taiwan; ${ }^{3}$ Institute of Clinical Medicine, National Yang-Ming University, Taipei, Taiwan; ${ }^{4}$ Institute of Traditional Medicine, \\ School of Medicine, National Yang-Ming University, Taipei, Taiwan \\ Contributions: (I) Conception and design: CS Lin, YM Kang; (II) Administrative support: CY Shiau, KL Lan, YM Liu; (III) Provision of study \\ materials or patients: CY Shiau, KL Lan, LW Wang, YM Liu, JC Lee; (IV) Collection and assembly of data: CS Lin, PI Huang, YW Hu, IC Lai, \\ YH Wu, TY Lai, WC Yang, JC Lee, YM Kang; (V) Data analysis and interpretation: All authors; (VI) Manuscript writing: All authors; (VII) Final \\ approval of manuscript: All authors. \\ Correspondence to: Cheng-Ying Shiau, MD. Division of Radiation Oncology, Department of Oncology, Taipei Veterans General Hospital, 201, Sec.2, \\ Shih-Pai Road, Taipei 11217, Taiwan. Email: cyshiau@vghtpe.gov.tw.
}

Background: Tenosynovial giant cell tumor (TGCT), historically known as pigmented villonodular synovitis (PVNS), is a rare and benign proliferative disease of joints. Diffuse form TGCT/PVNS has higher local recurrence rate that requires surgery and adjuvant treatment such as radiotherapy (RT). We reported our experience in treating patients with advanced TGCT/PVNS by surgery and RT or RT alone.

Methods: We included 48 patients of TGCT/PVNS who received RT between January 2001 and December 2018. Patients demographics, treatment parameters, complications and recurrences were evaluated. The Kaplan-Meier curves of local control (LC) were estimated, and variables affecting LC were analyzed by the univariate and multivariate Cox proportional hazard model.

Results: All patients had diffuse form TGCT/PVNS. Knee joint was affected in 34 patients (70.8\%). Eight patients (16.7\%) were inoperable so definitive RT was delivered. Most of the patients (85.4\%) received RT dose of $30 \mathrm{~Gy}$ in 15 fractions. The median follow-up time was 4.3 years, and seven patients (14.6\%) suffered from recurrence. Obesity (body mass index $>27 \mathrm{~kg} / \mathrm{m}^{2}$ ) was a significant prognostic factor for local recurrence in univariate and multivariate Cox regression analyses. The overall 1-, 3- and 5-year LC rate was $100 \%, 92.8 \%$ and $87.2 \%$, respectively. There was no significant surgical complication. RT-related acute toxicities were mild, including grade 1 dermatitis, joint effusion and decrease in joint range of motion.

Conclusions: RT in TGCT/PVNS patients revealed good LC and low treatment toxicities. Obesity was associated with higher recurrence rate. Definitive RT may be a treatment option for inoperable patients.

Keywords: Giant cell tumor of tendon sheath; obesity; radiotherapy (RT); synovitis, pigmented villonodular

Received: 24 May 2020; Accepted: 20 July 2020; Published: 30 September 2020.

doi: $10.21037 /$ tro-20-41

View this article at: http://dx.doi.org/10.21037/tro-20-41

^ ORCID: 0000-0002-1750-0736. 


\section{Introduction}

Tenosynovial giant cell tumor (TGCT), historically known as pigmented villonodular synovitis (PVNS), is a rare, benign proliferative disorder arising from synovial cells of joint capsules, bursae, and tendon sheaths most commonly affecting young adults. The estimated incidence of TGCT/PVNS is about 1.8 per million population (1). It is characterized histologically by the presence of inflammation, hemosiderin deposition, multinucleated giant cells, and lipid-laden macrophages (2). There is no consensus on the etiology and pathogenesis of the disease. Some risk factors have been recognized such as trauma, chronic inflammation, and abnormal lipid metabolism (3). Erosion of cartilage and bone will occur, causing deformity and limited range of motion in the affected joint if left untreated.

TGCT/PVNS is typically a monoarticular process that usually affects large joints. Its clinical course is slow and insidious onset of pain, swelling, stiffness and reduced range of motion in the involved joint (4). It occurs in two histopathologic forms: the diffuse form, and the localized form. The localized form is characterized by single, nodular or pedunculated masses surrounded by normal synovial tissue, while the diffuse form involves the entire synovium and accounts for the majority of cases (5). Magnetic resonance imaging (MRI) is key to establishing the correct diagnosis showing nodular synovial proliferation with low signal on T1- and T2-weighted images, secondary to hemosiderin deposition (6-8).

The first-line treatment of choice for all types of TGCT/ PVNS is radical resection of the involved tissue, but the risk of recurrence is high after the operation, especially in diffuse TGCT/PVNS $(9,10)$. Post-operative radiotherapy (RT) is not needed for localized TGCT/PVNS but it is associated with a reduced rate of recurrence for diffuse TGCT/PVNS $(11,12)$. The planned treatment volume includes the whole synovial space and eventually all invasive components of the disease as the synovial cells are the radiation target and an inhibition of its proliferation is suggested as RT mechanism $(9,13,14)$.

Due to small number of cases, studies in diffuse form TGCT/PVNS receiving RT are scarce. To the best of our knowledge, this is the first article to discuss the local control (LC) rate via Kaplan-Meier estimates and prognostic factors with a relatively large number of TGCT/PVNS patients. In this study, we retrospectively evaluated the treatment and outcome of TGCT/PVNS patients who received RT in our hospital. We present the following article in accordance with the STROBE reporting checklist (available at http:// dx.doi.org/10.21037/tro-20-41).

\section{Methods}

\section{Patient recruitment}

A retrospective study of orthopedic patients treated at Taipei Veterans General Hospital (VGHTPE) between January 2001 and December 2018 was performed by review of the electronic RT registry. The study was conducted in accordance with the Declaration of Helsinki (as revised in 2013). The study was approved by institutional ethics board of VGHTPE (NO.: 2020-06-006AC) and a request to waive of informed consent was approved because it was no more than minimal risk. Eligibility included: (I) clinical or pathological diagnosis of TGCT/PVNS, (II) receiving operation with adjuvant RT or definitive RT alone, (III) at least one year of follow-up after treatment. Table 1 summarizes the characteristics of the 48 patients recruited.

\section{Operation and $R T$}

For patients who received operations in our study, they received open synovectomy, arthroscopic synovectomy or both, depending on orthopedic surgeons' evaluation. Treatment was individualized according to patient characteristics and lesion extension. Adjuvant RT and definitive RT were delivered by linear accelerators with mega-voltage $\mathrm{X}$-rays $(4,6$, and $10 \mathrm{MV})$ using threedimensional conformal RT or intensity-modulated RT. The median dose of irradiation was 30 Gy (range, 30-40 Gy), in 15 daily fractions of 2 Gy. The clinical target volume of RT included whole synovial space with TGCT/PVNS involvement as well as the cartilage and bony structures invaded by proliferative tissue. An expansion of at least $0.5 \mathrm{~cm}$ was delineated as the planning target volume. An example of clinical target delineation and treatment planning was illustrated in Figure 1.

\section{Assessment of treatment results}

Patients were followed every 3 months in the first year, every 6 months in the second year and annually thereafter. Disease control was assessed by physical examination and MRI at 3, 6, 9, 12, 18 and 24 months and annually after treatment to at least 5 years in all patients. If associated 
Table 1 Demographic characteristics and treatment parameters

\begin{tabular}{|c|c|}
\hline Characteristic & Number of patients $(\mathrm{N}=48)$ \\
\hline \multicolumn{2}{|l|}{ Sex, n (\%) } \\
\hline Male & $19(39.6)$ \\
\hline Female & $29(60.4)$ \\
\hline Age (years), median [range] & $39[15-76]$ \\
\hline BMI $\left(\mathrm{kg} / \mathrm{m}^{2}\right)$, median [range] & $23.1[15.8-40.6]$ \\
\hline \multicolumn{2}{|l|}{ Site, n (\%) } \\
\hline Shoulder & $1(2.1)$ \\
\hline Elbow & $2(4.2)$ \\
\hline Hip & $4(8.3)$ \\
\hline Knee & $34(70.8)$ \\
\hline Ankle & $7(14.6)$ \\
\hline \multicolumn{2}{|l|}{ Laterality, n (\%) } \\
\hline Right & $25(52.1)$ \\
\hline Left & $23(47.9)$ \\
\hline Previous trauma, n (\%) & $6(12.5)$ \\
\hline \multicolumn{2}{|l|}{ Symptoms, n (\%) } \\
\hline Pain & $41(85.4)$ \\
\hline Swelling & $26(54.2)$ \\
\hline Stiffness & $4(8.3)$ \\
\hline \multicolumn{2}{|l|}{ No. of surgeries ${ }^{\dagger}, \mathrm{n}(\%)$} \\
\hline 0 & $29(60.4)$ \\
\hline 1 & $12(25.0)$ \\
\hline 2 & $7(14.6)$ \\
\hline \multicolumn{2}{|l|}{ Presenting status, n (\%) } \\
\hline Primary & $29(60.4)$ \\
\hline Recurrence & $19(39.6)$ \\
\hline \multicolumn{2}{|l|}{ RT type, n (\%) } \\
\hline Definitive & $8(16.7)$ \\
\hline Post-operative & $40(83.3)$ \\
\hline Deferred RT (>40 days) $)^{\ddagger}, \mathrm{n}(\%)$ & $27(56.3)$ \\
\hline \multicolumn{2}{|l|}{ RT dose, n (\%) } \\
\hline $30 \mathrm{~Gy} / 15 \mathrm{Fx}$ & $41(85.4)$ \\
\hline 36 Gy/18 Fx & $2(4.2)$ \\
\hline $40 \mathrm{~Gy} / 20 \mathrm{Fx}$ & $5(10.4)$ \\
\hline
\end{tabular}

${ }^{\dagger}$, number of surgeries in previous courses, excluding surgery in current course of treatment; ${ }^{\ddagger}$, deferred RT is defined as interval from surgery to adjuvant RT or from diagnosis to definitive RT $>40$ days. BMI, body mass index; RT, radiotherapy; Fx, fraction(s).
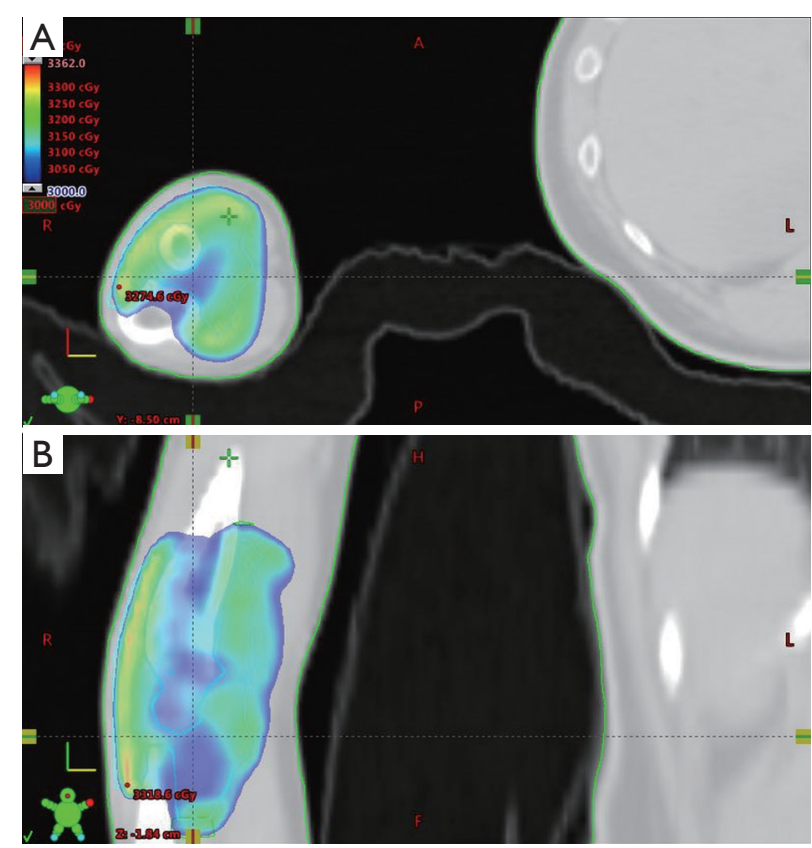

Figure 1 An example of radiotherapy planning of elbow tenosynovial giant cell tumor/pigmented villonodular synovitis with axial (A) and coronal (B) view. A cast was made to stabilize the patient position. Pre-operative magnetic resonance imaging was co-registered with image fusion to help clinical target volume delineation (light cyan line). Intensity-modulated radiotherapy with five portals were used to create conformal plan.

symptoms occurred, additional MRI would be arranged. Treatment results were retrospectively assessed by posttreatment MRI with clinical correlation. Recurrence was defined as the new appearance of a tumor lesion or enlargement of residual soft tissue in the primary site, assessed by experienced orthopedic radiologist. Persistent thickening of synovium without interval change was regarded as stable disease. Common Terminology Criteria for Adverse Events (version 4.03) was used to evaluate adverse treatment effects.

\section{Statistical analysis}

Descriptive statistics, including medians and proportions, were used to characterize the patients and treatment results. The Kaplan-Meier method was used to determine the $\mathrm{LC}$ rate. The duration of $\mathrm{LC}$ was defined as the time from the first date of intervention (operation or RT) to the date of any evidence of recurrence or the last follow-up. The Kaplan-Meier curves of LC with different variables 
were compared by log-rank test. Variables affecting LC were analyzed by the univariate and multivariate Cox proportional hazard model. All the analyses were performed using R project (version R-3.6.3; http://www.r-project. org). A $\mathrm{P}$ value less than 0.05 was considered statistically significant.

\section{Results}

\section{Baseline characteristics}

Twenty-nine women (60.4\%) and 19 men (39.6\%) were identified, giving a gender ratio (female/male) of 1.53 , with a median age of 39 years (range, 15-76 years). Among our patients, 46 patients $(95.8 \%)$ received pre-treatment evaluation via MRI, while only 2 patients (4.2\%) were determined by plain film and clinical findings. Fortyseven patients $(97.9 \%)$ had pathology confirmation of the disease. The knee joint was affected in 34 patients (70.8\%). All patients had diffuse form TGCT/PVNS. Six patients $(12.5 \%)$ reported a prior history of trauma to the involved joint. Most common clinical symptoms were pain (85.4\%), followed by swelling $(54.2 \%)$ and stiffness $(8.3 \%)$. Other baseline characteristics were summarized in Table 1.

\section{Treatment modalities}

A total of 19 patients (39.6\%) were treated as recurrent disease and had received one or two surgeries in previous courses of treatment. Eight patients $(16.7 \%)$ were not suitable for surgery because of probable function loss of the affected joint so definitive RT (dose 30-40 Gy) was delivered. Of the 40 patients having surgery in current course, 37 patients $(92.5 \%)$ were treated with open synovectomy while two patients $(5.0 \%)$ received arthroscopic synovectomy and one patient $(2.5 \%)$ underwent both. The interval between surgery and postoperative RT or diagnosis to definitive RT was greater than 40 days in 23 patients $(47.9 \%)$ and it was regarded as deferred RT. Most of the patients (85.4\%) received RT dose of $30 \mathrm{~Gy}$ in 15 fractions (Table 1). Higher dose was given in patients with high risk of recurrence or when gross residual tumor was highly suspected by the radiation oncologists.

\section{Outcomes and factors}

The median follow-up time was 4.3 years (range, $1.2-$ 16.4 years). Seven patients (14.6\%) had disease recurrence after RT and all of them were from the group of surgery followed by post-operative RT while patients receiving definitive RT were all free of recurrence. The median time of treatment to recurrence was 3.3 years (range, 1.7-6.9 years). Recurrences were treated by salvage operation alone in one patient, operation with adjuvant RT in one patient and close follow-up in five patients due to slow progression of symptoms.

In univariate Cox regression analysis, obesity [body mass index $(\mathrm{BMI})>27 \mathrm{~kg} / \mathrm{m}^{2}$ ] (hazard ratio $=4.61 ; 95 \%$ CI, $1.08-19.67 ; \mathrm{P}=0.039$ ) as well as ankle joint affected (hazard ratio $=4.46 ; 95 \% \mathrm{CI}, 1.08-18.42 ; \mathrm{P}=0.039$ ) were found to be significant prognostic factors for local recurrence (Table 2). Sex, disease status (primary or recurrence), timing of RT and RT dose were not significant factors for recurrence (Figure 2). However, only obesity remained significance in multivariate Cox proportional hazard analysis (hazard ratio $=5.32 ; 95 \%$ CI, $1.07-26.50 ; \mathrm{P}=0.041$ ) (Table 2).

The overall 1-, 3- and 5-year LC rate was 100\%, 92.8\% and $87.2 \%$, respectively (Figure 3). The obese group (BMI $>27 \mathrm{~kg} / \mathrm{m}^{2}$ ) had a 1 -, 3- and 5-year LC rate of $100 \%, 66.7 \%$ and $66.7 \%$ while the non-obese group had a 1-, 3- and 5 -year LC rate of $100 \%, 97.0 \%$ and $89.4 \%$, respectively (Figure 2B).

\section{Toxicities and compliance}

No significant surgical complication was found. In terms of RT-related acute toxicities, there were 12 patients $(25.0 \%)$ reporting grade 1 dermatitis, 11 patients (22.9\%) with grade 1 joint effusion and two patients (4.2\%) with grade 1 joint range of motion decrease. There was no interruption of RT and all the patients completed treatment by schedule. No late toxicity or secondary malignancy was found during the follow-up period. Total joint arthroplasty was required in five patients (10.4\%) because of symptomatic osteoarthritis. The median time of treatment to arthroplasty was 4.5 years (range, 0.9-11.7 years). None of the five patients had disease recurrence.

\section{Discussion}

In our study, we found that obesity (BMI $>27 \mathrm{~kg} / \mathrm{m}^{2}$ ) is a prognostic factor via multivariate Cox proportional hazard analysis. Possible explanation includes greater loading of weight-bearing joints as well as abnormal lipid metabolism which is suspected to be a possible etiology of TGCT/PVNS (15). Obesity is also associated with 
Table 2 The hazard ratio and $\mathrm{P}$ values of each factor in univariate and multivariate Cox model for disease recurrence

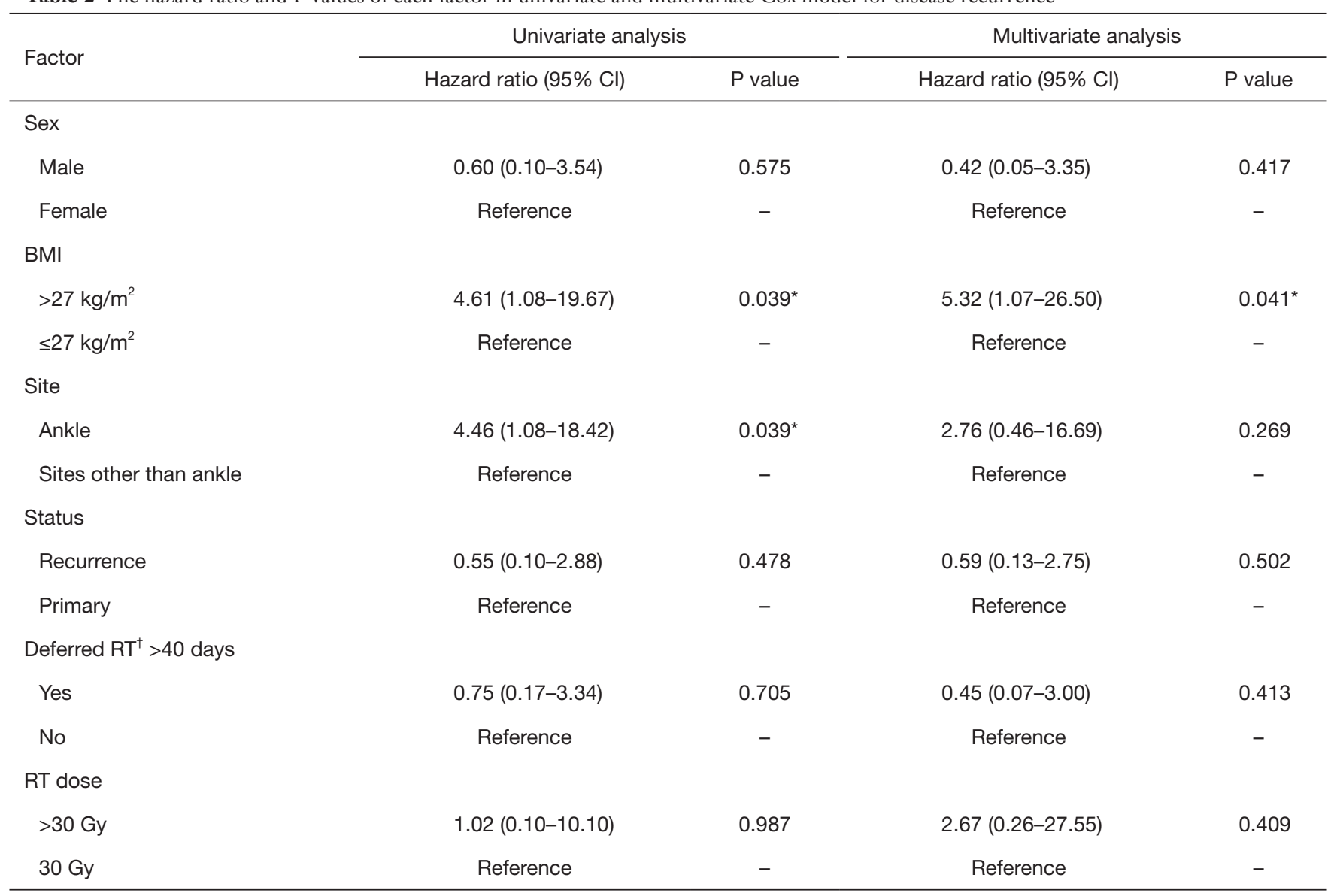

${ }^{\dagger}$, deferred RT is defined as interval from surgery to adjuvant RT or from diagnosis to definitive RT $>40$ days; *, P value $<0.05$. Cl, confidence interval. BMI, body mass index; RT, radiotherapy.

inflammation (16), and chronic inflammation has long been associated with TGCT/PVNS (2). The involvement of ankle joint was found to have inferior outcome comparing with other sites in univariate analysis and log-rank test but it turned out to be non-significant in multivariate Cox proportional hazard analysis. There is no study comparing prognosis between different joints so far. As the prognosis of TGCT/PVNS in different joints might vary from each other, further research is needed to find out the difference and develop proper treatment modalities for each site.

Since TGCT/PVNS is a rare disease (1), most studies discussed it with case reports or case series, and studies of treatment outcome after RT were seldom reported. Our study recruited substantial subjects representing a group of patients that required RT as adjuvant or definitive treatment. The Kaplan-Meier estimates give clear information on the recurrence rate over time. The radiation dose in our study was confirmed to attain balance between treatment effect and toxicities. We also found several interesting risk factors (obesity, ankle joint) via the univariate and multivariate Cox proportional hazard analysis which previous studies have not mentioned before.

The cohort we recruited were patients with diffuse form TGCT/PVNS with high risk of recurrence, either with bulky tumor, multiple recurrence or not suitable for surgery. Although RT has been reported to enhance LC after surgery, no randomized trials have proved this concept (17). The role of RT and the dose fractionation have not been well-established. The long-term radiation toxicities such as joint stiffness, tissue fibrosis, and radiation-associated secondary malignancies are major concerns in the treatment of this non-malignant disease. We used moderate dose RT to increase LC and minimize radiation-related toxicities. 
A
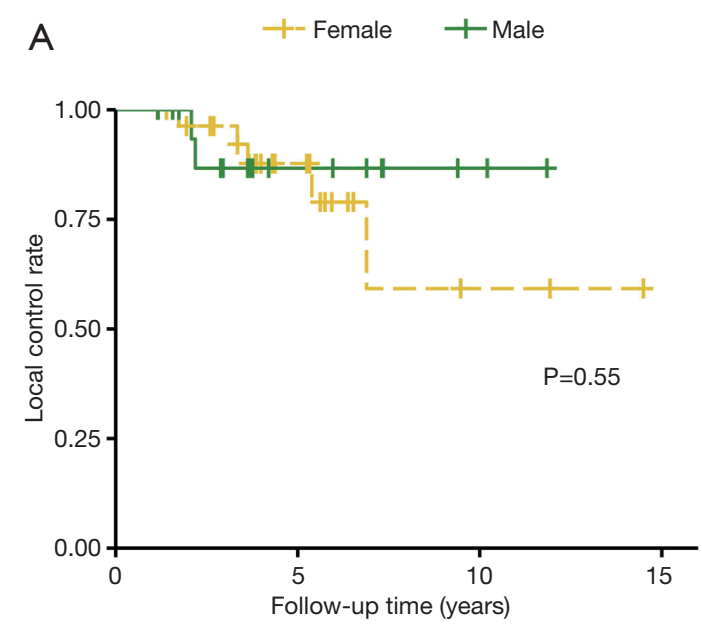

C

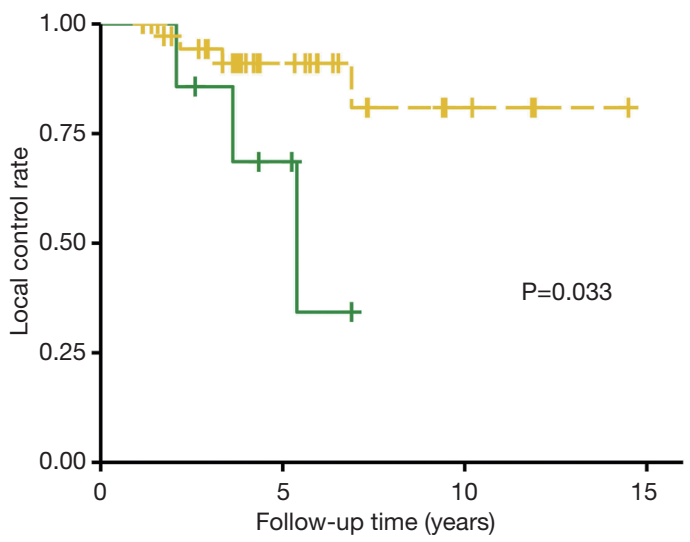

$\mathrm{E}$
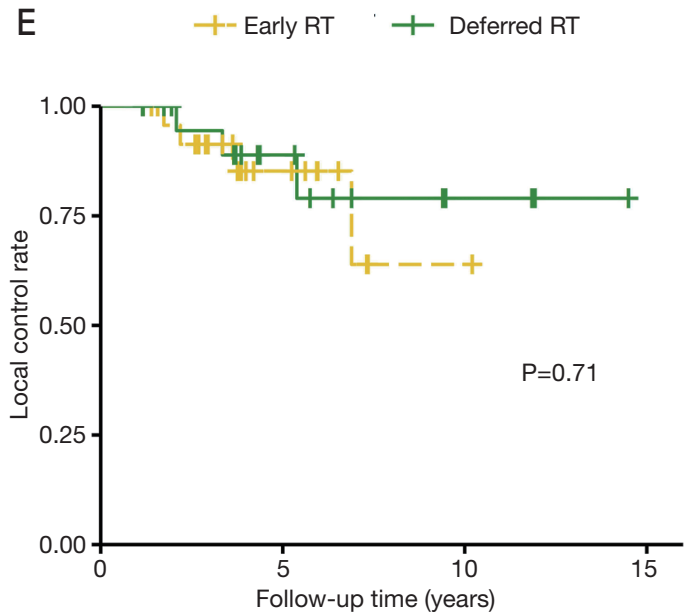

B

$$
+-\mathrm{BMI} \leq 27+\mathrm{BMI}>27
$$

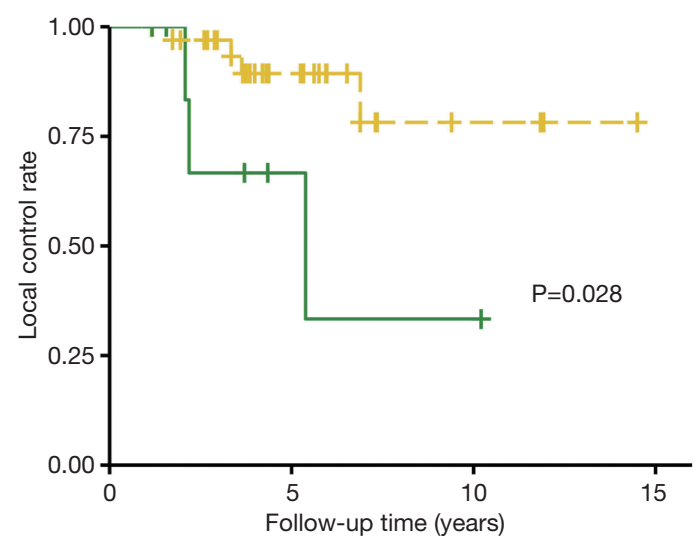

D

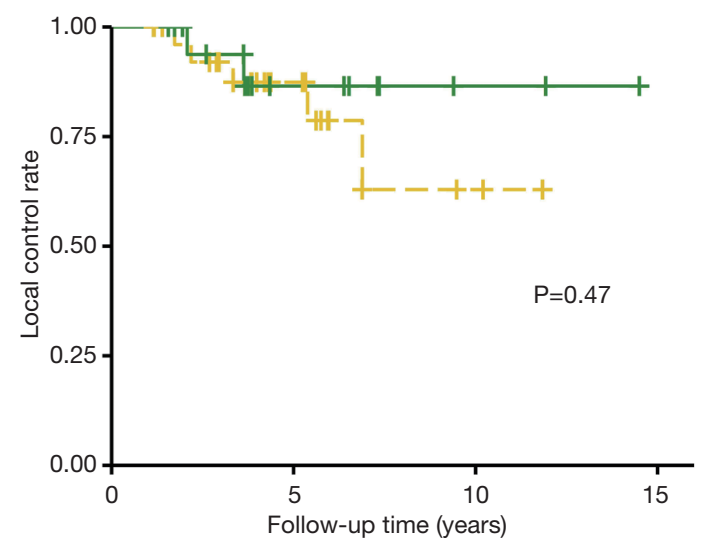

$\mathrm{F}$

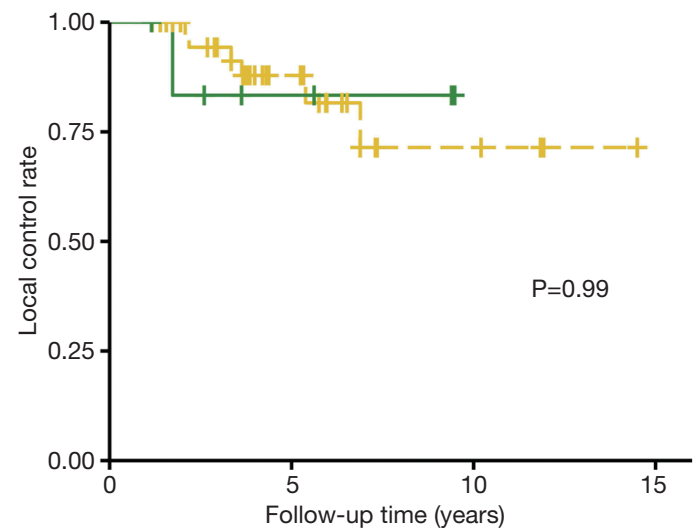

Figure 2 Kaplan-Meier curves of local control rate by sex (A), body mass index (BMI) $\left(\mathrm{kg} / \mathrm{m}^{2}\right)$ (B), sites (ankle or other sites) (C), status (primary or recurrence) (D), timing of radiotherapy (RT) (early or deferred) with cut-off value of 40 days from surgery to RT or diagnosis to definitive RT (E), and RT dose (F). The P values were calculated by log-rank test. 


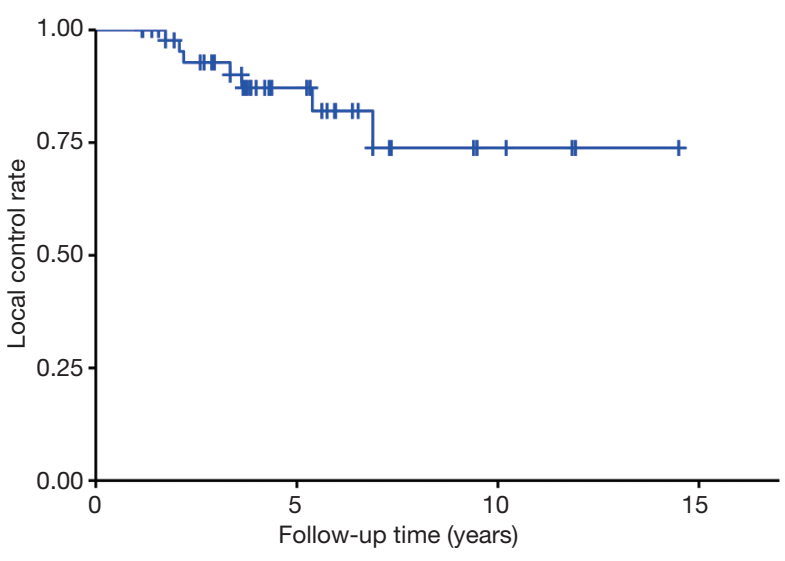

Figure 3 Kaplan-Meier curve of local control rate for all patients.

The outcomes of our study were consistent with historical data. A meta-analysis published in 2015 reported recurrence rate of $12.0 \%$ in the peri-operative RT group of diffuse TGCT/PVNS patients (11) which is compatible to our results with crude recurrence rate of $14.6 \%$ and 5 -year LC rate of $87.2 \%$. A study from German Cooperative Group on Radiotherapy in Benign Diseases revealed a dose range of 30-50 Gy (median, $36 \mathrm{~Gy}$ ) as adjuvant RT (18). Our data showed that 30 Gy in 15 fractions was sufficient for most of the patients and higher dose did not reduce the risk for recurrence.

It is challenging to manage patients with inoperable diffuse TGCT/PVNS. Eight patients who were unfit for surgery underwent definitive RT alone (dose 30-40 Gy). None of them had recurrence at the end of follow-up. Although the case number is small, moderate dose definitive RT seems to be an effective treatment option for inoperable patients. Such treatment modality may be investigated in the future. As for persistent or recurrent disease, further surgical intervention such as total synovectomy, subtotal resection, joint replacement, or amputation is recommended first. Since TGCT/PVNS is a non-fatal disease, functional outcome, and quality of life after surgery should be considered carefully. Another treatment of choice for this population is colony stimulating factor 1 (CSF1) receptor inhibitors such as pexidartinib, nilotinib, emactuzumab which have been studied in clinical studies (19-21). In TGCT/PVNS, a translocation in CSF1-COL6A3, $\mathrm{t}(1 ; 2)$ (p13;q35) causes overexpression of CSF1, attracting nonneoplastic cells which express CSF1 receptor (22). The accumulation of those non-neoplastic cells creates tumorous mass. Inhibition of CSF1 receptor by pexidartinib has shown robust response in TGCT/PVNS cases not amenable to improvement with surgery in a phase 3 trial (21). However, those target agents were not available in our institute.

Our study has several limitations. Firstly, the number of the participants is not large enough that association between some factors and outcome may be obscured. However, it is a rare disease and we still manage to find significant factors from our cases. Secondly, we didn't obtain the data of inflammatory markers such as C-reactive protein and neutrophil-lymphocyte ratio which were found to be significant predictors in a previous study (23). Thirdly, absence of quality-of-life information and functional score evaluation makes it difficult to present a comprehensive outcome of our treatment. In addition, the follow-up time is not long enough for late recurrence and radiation-induced secondary malignancy.

In conclusion, our results revealed good LC and low treatment toxicities in patients with TGCT/PVNS who received RT. Obesity $\left(\mathrm{BMI}>27 \mathrm{~kg} / \mathrm{m}^{2}\right.$ ) was associated with higher recurrence rate among these patients. Definitive RT may be a treatment option for inoperable patients.

\section{Acknowledgments}

We would like to express our gratitude to all the members in Department of Oncology and Orthopedic Surgery in Taipei Veterans General Hospital.

Funding: None.

\section{Footnote}

Reporting Checklist: The authors have completed the STROBE reporting checklist. Available at http://dx.doi. org/10.21037/tro-20-41

Conflicts of Interest: All authors have completed the ICMJE uniform disclosure form (available at http://dx.doi. org/10.21037/tro-20-41). LWW serves as an unpaid editorial board member of Therapeutic Radiology and Oncology from May 2020 to Apr 2022. YML serves as an unpaid editorial board member of Therapeutic Radiology and Oncology from Jun 2020 to May 2022. The other authors have no conflicts of interest to declare.

Ethical Statement: The authors are accountable for all aspects of the work in ensuring that questions related 
to the accuracy or integrity of any part of the work are appropriately investigated and resolved. The study was conducted in accordance with the Declaration of Helsinki (as revised in 2013). The study was approved by institutional ethics board of VGHTPE (NO.: 2020-06-006AC) and a request to waive of informed consent was approved because it was no more than minimal risk.

Open Access Statement: This is an Open Access article distributed in accordance with the Creative Commons Attribution-NonCommercial-NoDerivs 4.0 International License (CC BY-NC-ND 4.0), which permits the noncommercial replication and distribution of the article with the strict proviso that no changes or edits are made and the original work is properly cited (including links to both the formal publication through the relevant DOI and the license). See: https://creativecommons.org/licenses/by-nc-nd/4.0/.

\section{References}

1. Myers BW, Masi AT. Pigmented villonodular synovitis and tenosynovitis: a clinical epidemiologic study of 166 cases and literature review. Medicine (Baltimore) 1980;59:223-38.

2. Goldman AB, DiCarlo EF. Pigmented villonodular synovitis. Diagnosis and differential diagnosis. Radiol Clin North Am 1988;26:1327-47.

3. Ottaviani S, Ayral X, Dougados M, et al. Pigmented villonodular synovitis: a retrospective single-center study of 122 cases and review of the literature. Semin Arthritis Rheum 2011;40:539-46.

4. Gelhorn HL, Tong S, McQuarrie K, et al. Patientreported Symptoms of Tenosynovial Giant Cell Tumors. Clin Ther 2016;38:778-93.

5. Abdul-Karim FW, el-Naggar AK, Joyce MJ, et al. Diffuse and localized tenosynovial giant cell tumor and pigmented villonodular synovitis: a clinicopathologic and flow cytometric DNA analysis. Hum Pathol 1992;23:729-35.

6. Bhimani MA, Wenz JF, Frassica FJ. Pigmented villonodular synovitis: keys to early diagnosis. Clin Orthop Relat Res 2001;(386):197-202.

7. Cheng XG, You YH, Liu W, et al. MRI features of pigmented villonodular synovitis (PVNS). Clin Rheumatol 2004;23:31-4.

8. Dürr HR, Stäbler A, Maier M, et al. Pigmented villonodular synovitis. Review of 20 cases. J Rheumatol 2001;28:1620-30.

9. Seegenschmiedt MH, Micke O, Niewald M, et al. DEGRO guidelines for the radiotherapy of non- malignant disorders: part III: hyperproliferative disorders. Strahlenther Onkol 2015;191:541-8.

10. Korim MT, Clarke DR, Allen PE, et al. Clinical and oncological outcomes after surgical excision of pigmented villonodular synovitis at the foot and ankle. Foot Ankle Surg 2014;20:130-4.

11. Mollon B, Lee A, Busse JW, et al. The effect of surgical synovectomy and radiotherapy on the rate of recurrence of pigmented villonodular synovitis of the knee: an individual patient meta-analysis. Bone Joint J 2015;97-B:550-7.

12. Guo Q, Shi W, Jiao C, et al. Results and recurrence of pigmented villonodular synovitis of the ankle: does diffuse PVNS with extra-articular extension tend to recur more often? Knee Surg Sports Traumatol Arthrosc 2018;26:3118-23.

13. Heyd R, Seegenschmiedt MH, Micke O. The role of external beam radiation therapy in the adjuvant treatment of pigmented villonodular synovitis. Z Orthop Unfall 2011;149:677-82.

14. Duan Y, Qian J, Chen K, et al. Necessity of adjuvant postoperative radiotherapy for diffuse pigmented villonodular synovitis of the knee: A case report and literature review. Medicine (Baltimore) 2018;97:e9637.

15. Steinmetz S, Rougemont AL, Peter R. Pigmented villonodular synovitis of the hip. EFORT Open Rev 2017;1:260-6.

16. Karczewski J, Śledzińska E, Baturo A, et al. Obesity and inflammation. Eur Cytokine Netw 2018;29:83-94.

17. Griffin AM, Ferguson PC, Catton CN, et al. Longterm outcome of the treatment of high-risk tenosynovial giant cell tumor/pigmented villonodular synovitis with radiotherapy and surgery. Cancer 2012;118:4901-9.

18. Heyd R, Micke O, Berger B, et al. Radiation therapy for treatment of pigmented villonodular synovitis: results of a national patterns of care study. Int J Radiat Oncol Biol Phys 2010;78:199-204.

19. Cassier PA, Italiano A, Gomez-Roca CA, et al. CSF1R inhibition with emactuzumab in locally advanced diffusetype tenosynovial giant cell tumours of the soft tissue: a dose-escalation and dose-expansion phase 1 study. Lancet Oncol 2015;16:949-56.

20. Gelderblom H, Cropet C, Chevreau C, et al. Nilotinib in locally advanced pigmented villonodular synovitis: a multicentre, open-label, single-arm, phase 2 trial. Lancet Oncol 2018;19:639-48.

21. Tap WD, Gelderblom H, Palmerini E, et al. Pexidartinib versus placebo for advanced tenosynovial giant cell tumour (ENLIVEN): a randomised phase 3 trial. Lancet 
2019;394:478-87.

22. West RB, Rubin BP, Miller MA, et al. A landscape effect in tenosynovial giant-cell tumor from activation of CSF1 expression by a translocation in a minority of tumor cells. Proc Natl Acad Sci U S A 2006;103:690-5.

doi: $10.21037 /$ tro-20-41

Cite this article as: Lin CS, Kang YM, Lan KL, Wang LW, Liu YM, Huang PI, Hu YW, Lai IC, Wu YH, Lai TY, Yang WC, Lee JC, Shiau CY. Treatment and outcome of tenosynovial giant cell tumor/pigmented villonodular synovitis patients receiving radiotherapy in Taiwan: a single-center experience. Ther Radiol Oncol 2020;4:14.
23. Zhao G, Wang J, Xia J, et al. The predictive value of preoperative neutrophil-lymphocyte ratio (NLR) on the recurrence of the local pigmented villonodular synovitis of the knee joint. BMC Musculoskelet Disord 2018;19:339. 